\title{
DO SUBMILLIMETER GALAXIES REALLY TRACE THE MOST MASSIVE DARK-MATTER HALOS? DISCOVERY OF A HIGH-z CLUSTER IN A HIGHLY ACTIVE PHASE OF EVOLUTION
}

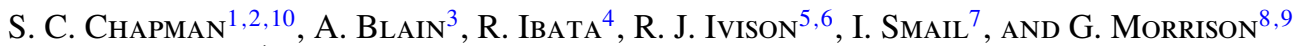 \\ ${ }^{1}$ Institute of Astronomy, Madingley Road, Cambridge CB3 0HA, UK \\ 2 Department of Physics and Astronomy, University of Victoria, Victoria, B.C. V8P 1A1, Canada \\ 3 California Institute of Technology, Pasadena, CA 91125, USA \\ ${ }^{4}$ Observatoire de Strasbourg, 11, rue de l'Université, F-67000 Strasbourg, France \\ ${ }^{5}$ UK Astronomy Technology Centre, Royal Observatory, Blackford Hill, Edinburgh EH9 3HJ, UK \\ ${ }^{6}$ Institute for Astronomy, University of Edinburgh, Blackford Hill, Edinburgh EH9 3HJ, UK \\ ${ }^{7}$ Institute for Computational Cosmology, Durham University, South Road, Durham DH1 3LE, UK \\ ${ }^{8}$ Institute for Astronomy, University of Hawaii, Honolulu, HI 96822, USA \\ ${ }^{9}$ Canada-France-Hawaii Telescope, Kamuela, HI 96743, USA \\ Received 2008 June 13; accepted 2008 September 8; published 2009 January 16
}

\begin{abstract}
We present detailed observations of a $z \sim 1.99$ cluster of submillimeter galaxies (SMGs), discovered as the strongest redshift spike in our entire survey of $\sim 100 \mathrm{SMGs}$ across $800 \mathrm{arcmin}^{2}$. It is the largest blank-field SMG concentration currently known and has $<0.01 \%$ chance of being drawn from the underlying selection function for SMGs. We have compared UV observations of galaxies at this redshift, where we find a much less dramatic overdensity, having an $11 \%$ chance of being drawn from its selection function. We use this $z \sim 1.99$ overdensity to compare the biasing of UV- and submillimeter-selected galaxies, and test whether SMGs could reside in less overdense environments, with their apparent clustering signal being dominated by highly active merger periods in modest mass structures. We discuss the probable mechanisms for the apparently different bias we see at the two wavelengths. This impressively active formation phase in a low-mass cluster is not something seen in simulations, although we propose a toy model using merger bias, which could account for the bias seen in the SMGs. While enhanced buildup of stellar mass appears characteristic of other high- $z$ galaxy clusters, neither the UV galaxies nor SMGs in this structure exhibit larger stellar masses than their field galaxy counterparts (although the excess of SMGs in the structure represents a larger volume-averaged stellar mass than the field). Our findings have strong implications for future surveys of high- $z$ galaxies at long wavelengths such as SCUBA2 and Herschel. We suggest that since these surveys will select galaxies during their episodes of peak starbursts, they could probe a much wider range of environments than just the progenitors of rich clusters, revealing more completely the key events and stages in galaxy formation and assembly.
\end{abstract}

Key words: galaxies: clusters: general - galaxies: evolution - galaxies: high-redshift - galaxies: starburst large-scale structure of Universe

Online-only material: color figures

\section{INTRODUCTION}

Submillimeter galaxies (SMGs) have provided an efficient probe of ultraluminous $\left(>10^{12} L_{\odot}\right)$ star formation activity in the distant universe (e.g., Smail et al. 1997; Chapman et al. 2000; Blain et al. 2002; Borys et al. 2003; Coppin et al. 2008; Knudsen et al. 2008), with the bright submillimeter detection (rest-frame far-infrared (FIR)) providing an unambiguous signal of large dust masses heated predominantly by young stars rather than active galactic nuclei (AGNs; e.g., Almaini et al. 1999; Fabian et al. 2000; Alexander et al. 2005; Menendez-Delmestre et al. 2007; Pope et al. 2008). Piecing together the detailed properties of SMGs has been difficult because of the inherent dust obscuration to the regions of the largest bolometric output. High spatial resolution radio observations using the MERLIN interferometer (Chapman et al. 2004a; Biggs \& Ivison 2008) provided early indications that the dust and gas in SMGs was more compact than typical galaxies, yet still resolved on scales three times larger than in local ULIRGs. Once verified directly through high-resolution $\mathrm{CO}$ gas studies of SMGs (Tacconi et al. 2006, 2008) this was used to argue that SMGs are scaled up ULIRGs. This radio and CO mapping of SMGs has

\footnotetext{
${ }^{10}$ Canadian Space Agency Fellow.
}

suggested that the locus of FIR emission is often offset from UV/optical emission, consistent with findings (Chapman et al. 2005, hereafter C05) that even dust-corrected UV estimates of star formation rates (SFRs) in SMGs underpredict the true SFRs by factors $>100$ times on average. CO linewidths for a sizable sample of SMGs (Frayer et al. 1998; Neri et al. 2003; Greve et al. 2005) have suggested large dynamical masses, > $10^{11} M_{\odot}$, though not nearly as large as the halos in which they reside based on their clustering properties (Blain et al. 2004). The large dynamical masses are associated with large $\left(10^{11}\right.$ $2 \times 10^{11} M_{\odot}$ ) stellar masses (Borys et al. 2005; Hainline 2008; Swinbank et al. 2008). Finally, the gas masses and SFRs define an average gas exhaustion timescale $<50 \mathrm{Myr}$, suggesting a duty cycle of $\sim 10$ for an underlying population of galaxies hosting the submillimeter-luminous events. These studies bring together a picture of SMGs as typically representing massive, gas-rich galaxies, which ought to be building elliptical galaxies that are found preferentially in clusters at the present day (Swinbank et al. 2006, 2008).

The strongly clustered populations of red $K$-selected galaxies at $z=2-4$ (e.g., Daddi et al. 2003) and of luminous high$z$ radio galaxies (HzRG; Brand et al. 2005) suggest that they are formed in the highest density perturbations at early epochs, implying that they are the progenitors of local massive early- 
type galaxies and $z \sim 1$ extremely red objects (EROs), which themselves have similar strong clustering (e.g., Brown et al. 2005). HzRGs are believed to host massive black holes; thus may represent some of the most massive galaxies at these epochs (e.g., de Breuck et al. 2000) and could trace some of the highest density environments. The identification of modest excesses of companion galaxies around HzRGs in the optical/near-infrared (e.g., Rottgering et al. 1996), X-ray (Pentericci et al. 2002; Smail et al. 2003a), and submillimeter (Ivison et al. 2000; Stevens et al. 2003), supports their association with overdense structures.

Studies to date have suggested that the most FIR-luminous, distant galaxies are also clustered very strongly (Blain et al. 2004; Borys et al. 2004; Scott et al. 2002, 2006; van Kampen et al. 2005; Farrah et al. 2006; Blake et al. 2006; Gilli et al. 2007). At face value this would indicate that they occupy the largest dark-matter halos $\left(\sim 10^{13} M_{\odot}\right)$. However selection of galaxies by the most FIR-luminous specimens may be prone to finding the brief periods when systems of lower matter overdensity are rapidly evolving (e.g., Scannapieco \& Thacker 2003; Furlanetto \& Kamionkowski 2006). Indeed burst timescales as short as 10 Myr have been estimated for some SMGs (e.g., Smail et al. 2003b). Blain et al. (2004) calculated that, complex biases aside, SMGs have a clustering length that is consistent with a form of evolution ensuring their properties subsequently match the clustering length typical of evolved red galaxies at $z \sim 1$ and finally of rich clusters of galaxies at the present epoch.

However, as pointed out by Blain et al. (2004), this is inconsistent with the expected density of rich clusters. It is very unlikely $(0.05 \%$ chance $)$ that any field of order $10^{\prime} \times 10^{\prime}$ includes the progenitor of a moderately rich cluster (volume density $\rho=10^{-7} \mathrm{Mpc}^{-3}$ ), even in the long pencil beam from $z=1-3$ of a radio-SMG survey. This apparent paradox can only be resolved by reducing the frequency of such associations by a factor of $10\left(\rho=10^{-7} \mathrm{Mpc}^{-3}\right.$ observed for moderately rich clusters compared with $10^{-6} \mathrm{Mpc}^{-3}$ implied by SMGs). Blain et al. concluded that either the dark-matter halo masses are less extreme than inferred from a picture of simple 1-1 biasing at a given mass, or some other biasing effect must be at work. Adelberger et al. (2005b) presented an alternative explanation in the inherent bias of the cluster calculation methodology, however statistical analyses of the SMG clustering through other methods is consistent with the Blain et al. (2004) calculation (e.g., Blake et al. 2006). The alternative explanation, that SMGs often reside in less overdense environments (and that ULIRG clustering could be dominated by the coordination of highly active periods across modest mass environments), is tested here, using UV observations of the richest overdensity found in Blain et al. (2004) and Chapman et al. (2005).

In Section 2, we describe the sample properties, with Section 2.1 assessing the various selection functions, and developing a statistically robust measure of the galaxy overdensity. In Section 2.2, we analyze the properties of the galaxies lying in the overdense structure, including their SFRs at a number of wavelengths, their luminosity function (LF), and their angular positions on the sky. In Section 3, we discuss the theoretical expectations of the measured redshift space contrasts, propose an explanation for the differential bias seen at different wavelengths (Section 3.1), and discuss the implications for future surveys at submillimeter wavelengths (Section 3.2). We assume a $\Lambda$-cold dark matter (CDM) cosmology (Spergel et al. 2003) with $\Omega_{0}=0.3, \Omega_{\Lambda}=0.7$, and $H_{0}=70 \mathrm{~km} \mathrm{~s}^{-1} \mathrm{Mpc}^{-1}$, so that 1 arcsec corresponds to $8.4 \mathrm{kpc}$ physical size at $z=2.0$.

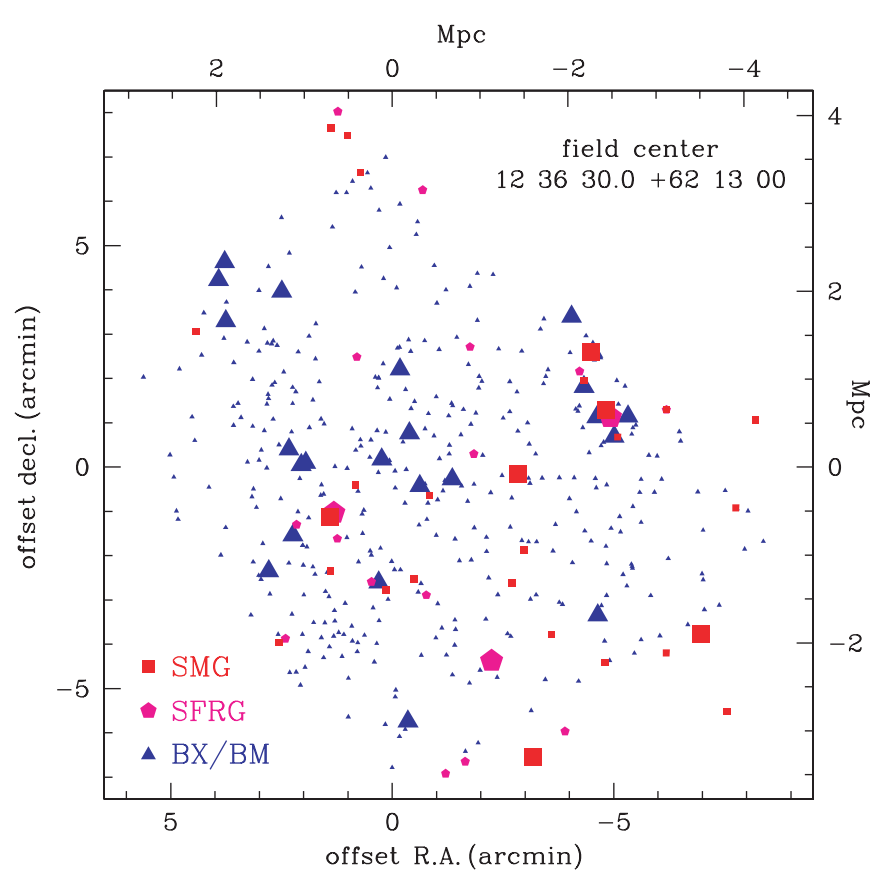

Figure 1. Positions of all UV-selected galaxies and SMGs/SFRGs in the survey region are shown as small symbols, while those lying in the $z=1.99$ structure are large symbols. Offset-projected Mpc are shown on the axes for reference at $z=2$. Galaxies in the $z=1.99$ structure are generally widely separated on the sky, with >Mpc separations between most SMGs/SFRGs except for two close ( $\sim 100 \mathrm{kpc})$ SMG/SFRG pairs in two cases.

(A color version of this figure is available in the online journal.)

\section{CHARACTERIZING THE STRUCTURE AT UV AND SUBMILLIMETER WAVELENGTHS}

Blain et al. (2004) highlighted an association of five SMGs in the GOODS-N field, all lying within $1200 \mathrm{~km} \mathrm{~s}^{-1}$ of $z=1.995$, representing the strongest association in their spectroscopic survey of 73 SMGs. The GOODS-N field is one of the better sampled fields from the expanded C05 SMG spectroscopic survey and the ongoing spectroscopic surveys of the SHADES submillimeter fields (e.g., Coppin et al. 2008; A. W. Blain et al. 2009, in preparation), however associations of similar significance can be reasonably ruled out in these other survey fields (although the association distribution function $N\left(n_{\mathrm{SMG}}\right)$ is not yet well characterized). To further study this GOODS-N field SMG association and its properties at other wavelengths, we bring together an expanded sample of SMGs in this field, representing our primary sample of ultraluminous star-forming galaxies at high redshift. A population of submillimeter-faint, radio-selected galaxies (SFRGs) with similar inferred SFRs to SMGs as evidenced by radio and $24 \mu \mathrm{m}$ luminosities (Chapman et al. 2004b; C. Casey et al. 2009, in preparation) represents a secondary sample from which to study the cluster and test our hypotheses. A map of source positions is shown in Figure 1, depicting the spatial distributions of all galaxies with known spectroscopic redshift $z>1$ in the field and highlighting the candidate $z=1.99$ structure.

The GOODS-N sample of 30 SMGs comprises the spectroscopic redshift sample of 22 radio-identified SMGs in Chapman et al. (2005), six additional radio-SMGs with spectroscopic redshifts presented in Pope et al. $(2006,2008)$, and two further radio-SMGs from Daddi et al. (2008). We also include 19 SFRGs from Chapman et al. (2004b) and C. Casey et al. (2009, in preparation), and assemble a complete catalog of UV/ 

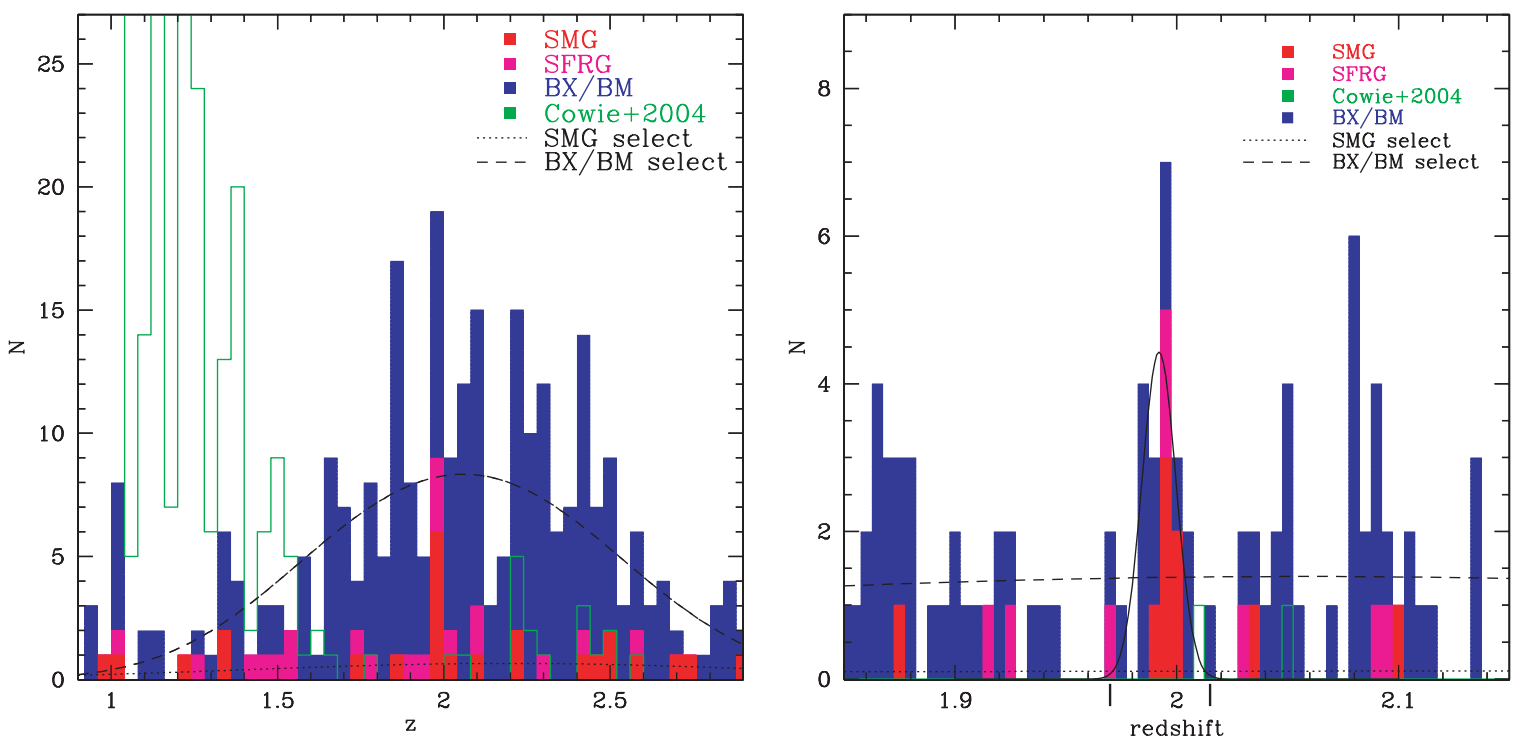

Figure 2. Left panel: redshift histograms in GOODS-N (bins of $\delta z=0.02$ ) from the surveys of Reddy et al. (2006) in UV-selected BX/BM galaxies, Team Keck Redshift Survey (Wirth et al. 2004), and Hawaii redshift survey (Cowie et al. 2004), the SMG surveys of Chapman et al. (2003, 2005), and the SFRG surveys of Chapman et al. (2004) and Casey et al. (2008). The $z=1.99$ structure can be seen as the highest peak of galaxies in the $z \sim 2$ region. The selection functions for SMGs and BX/BMs are depicted normalized to the number of sources in each sample. The SFRGs are included in the SMG selection function (see the text). There are a few overlapping BX/BM, TKRS, and SMGs/SFRGs that are effectively double-counted in the plot. Note that the BX and SMG/OFRG galaxy samples in the $z=1.99$ structure are completely nonoverlapping, and the structure could arguably be more significant than other BX galaxy peaks in the combined BX/SMG galaxy sample. Right panel: a zoomed-in view of the structure with smaller redshift bins $(\delta z=0.005)$. The spike galaxies listed in Table 1 are shown by the vertical bars.

(A color version of this figure is available in the online journal.)

optical-selected galaxies with redshifts, combining the surveys of Wirth et al. (2004), Cowie et al. (2004), and Reddy et al. (2006) with our own spectroscopic data. In Table 1, we list all known galaxies in the $z=1.99$ structure, including the several unpublished UV-selected galaxies from our surveys. We assemble the relevant archival multiwavelength data in Table 1, which is discussed explicitly in this paper, as well as new $1.4 \mathrm{GHz}$ very large array (VLA) radio measurements from the maps of Morrison et al. (2008). The redshift distributions are shown in Figure 2. The well-studied GOODS-N region is covered by a superb multiwavelength survey up to great sensitivity in most wavebands, and so it is unlikely that further missing galaxy populations could skew our results significantly. However, there is no spectroscopic sample available, which effectively targets quiescent galaxies at $z \sim 2$.

\subsection{Significance of the Structure}

There is an obvious $z \sim 1.99$ galaxy concentration in the SMG redshift distribution lying in the GOODS-N field, which coincides with an apparently less significant concentration in the UV-selected galaxies. Figure 1 shows the angular distribution of the galaxies, revealing that the nine SMGs and SFRGs in a redshift spike are not obviously clustered spatially across the field shown in Figure 1, spanning a region of $7^{\prime}(3.5 \mathrm{Mpc})$ on a side, although there are two relatively close pairs (of $9^{\prime \prime}$ and $17^{\prime \prime}, 76 \mathrm{kpc}$ and $143 \mathrm{kpc}$, respectively). The redshift distributions around the $z \sim 2$ spike are compared in Figure 2.

We first aim to characterize this structure with respect to galaxies selected at both UV and submillimeter/radio wavelengths. For the SMG sample, we define a selection function by smoothing the redshift distribution of the $\sim 100$ SMGs in the expanded survey from C05 described above. We adopt a cluster finding algorithm based on an Erlangian distribution function (Eadie et al. 1971),

$$
p(\Delta z \mid N \lambda)=\lambda(\lambda \Delta z)^{N-2} \exp (-\lambda \Delta z) /(N-2) !,
$$

which characterizes the probability (in the absence of clustering) that a group of $N$ galaxies would span a redshift interval $\Delta z$, considering that the product of our selection function and the density of SMGs is $\lambda$. The procedure is very similar to that described in Steidel et al. (1998), and was used for our studies of halo substructures in the Andromeda galaxy (e.g., Chapman et al. 2006).

First, looking just at the six SMGs in the structure, we find a probability of $0.005 \%$ for the concentration of SMGs at $\langle z\rangle=1.992$ with $\Delta z=0.008$ to be drawn from the selection function by chance, which maximizes the contrast with a peak of five out of the six SMGs lying within the region. We define a galaxy overdensity as the number of galaxies found in the structure (excluding the two galaxies which define the edges of the redshift interval) divided by the expected number in this redshift interval from the selection function (which is only 0.4), yielding $\delta_{g}^{z}=4 / 0.4=10$. This result is obviously dependent on the precise redshift adopted, as some scatter is introduced when individual galaxies are defined by different combinations of lines from molecular, neutral, and ionized gas, which can differ by as much as $1000 \mathrm{~km} \mathrm{~s}^{-1}$ in a given SMG (Chapman et al. 2005; Greve et al. 2005; Swinbank et al. 2004). If the SMGs and SFRGs are considered together, the contrast is maximized with seven of the nine galaxies, and the probability to find the structure by chance rises marginally to $0.007 \%$, with an implied overdensity $\delta_{g}^{z}=8$.

Second, for the UV-selected galaxies, we adopt the selection function defined by the smoothed distribution of all galaxies in the spectroscopic survey of Steidel et al. (2004) and Reddy et al. (2006), see Figure 1. The probability of the $z \sim 1.99$ concentration being drawn at random from the selection function is $11.3 \%$, and this contrast is maximized with a peak of 15 galaxies in the $\langle z\rangle=1.987, \Delta z=0.015$ region, with an overdensity 
Table 1

Properties of All UV-Selected Galaxies, SMGs, and SFRGs in GOODS-N $z=1.99$ Spike $^{\mathrm{a}}$

\begin{tabular}{|c|c|c|c|c|c|c|c|c|}
\hline ID & R.A. & Decl. & Redshift $^{\mathrm{b}}$ & Type of Redshift & $S_{1.4 \mathrm{GHz}}{ }^{\mathrm{c}}$ & $S_{24 \mu \mathrm{m}}$ & $M_{\mathrm{AB}, 5.8 \mu \mathrm{m}}$ & $M_{\mathrm{AB}, 4.5 \mu \mathrm{m}}$ \\
\hline SMG-93 & 123600.13 & +621047.2 & 1.994 & $\mathrm{UV}, \mathrm{H} \alpha$ & $128.5 \pm 8.1(26.5)$ & $1237 \pm 8.0$ & $20.32 \pm 0.27$ & $20.97 \pm 0.08$ \\
\hline SMG-132 & 123618.32 & +62 1550.5 & 1.999 & $\mathrm{CO}(4-3)$ & $172.0 \pm 8.4(34.3)$ & $344.0 \pm 8.0$ & $20.31 \pm 0.18$ & $20.65 \pm 0.07$ \\
\hline SMG-140e & 123621.25 & +621708.3 & 1.994 & Spitzer & $169.4 \pm 8.8(31.9)$ & $370.0 \pm 11.4$ & $20.06 \pm 0.10$ & $20.31 \pm 0.06$ \\
\hline SMG-140w & 123620.99 & +621709.5 & $1.989 / 1.988$ & $\mathrm{UV}, \mathrm{H} \alpha$ & $63.4 \pm 10.6(9.0)$ & $\ldots{ }^{d}$ & $20.67 \pm 0.09$ & $20.96 \pm 0.07$ \\
\hline SMG-169 & 123632.53 & +620759.7 & 1.993 & UV & $80.4 \pm 8.6(15.1)$ & $820.8 \pm 15.2$ & $19.64 \pm 0.15$ & $20.29 \pm 0.07$ \\
\hline SMG-172e & 123635.57 & +62 1424.0 & 2.001 & UV & $77.0 \pm 7.8(16.3)$ & $1410.0 \pm 13.9$ & $18.29 \pm 0.09$ & $18.90 \pm 0.06$ \\
\hline SMG-255 & 123711.99 & +621325.6 & 1.992 & UV, CO & $50.2 \pm 8.1(10.2)$ & $225.0 \pm 7.0$ & $21.79 \pm 0.21$ & $21.45 \pm 0.09$ \\
\hline SFRG-130 & 123617.54 & +621540.7 & 1.993 & UV & $234.3 \pm 8.6(45.1)$ & $20.2 \pm 5.2$ & $20.77 \pm 0.17$ & $20.58 \pm 0.08$ \\
\hline SFRG-179 & 123640.73 & +621011.0 & $1.968 / 1.977$ & UV & $72.5 \pm 8.3(13.7)$ & $83.0 \pm 7.8$ & $21.29 \pm 0.19$ & $21.33 \pm 0.07$ \\
\hline SFRG-254 & 123711.32 & +62 1330.9 & $1.996 / 1.993$ & $\mathrm{H} \alpha / \mathrm{CO}(4-3)$ & $127.4 \pm 8.7(23.0)$ & $537.3 \pm 9.1$ & $19.72 \pm 0.11$ & $20.01 \pm 0.06$ \\
\hline BX-1201 & 123614.13 & +621541.8 & $\ldots / 2.000$ & UV & $10.6 \pm 7.0(2.8)$ & $29.0 \pm 5.5$ & $23.04 \pm 0.27$ & $22.99 \pm 0.07$ \\
\hline BX-1192 & 123616.83 & +621514.3 & $\ldots / 1.996$ & UV & $21.3 \pm 10.3$ & $111.6 \pm 10.7$ & $21.47 \pm 0.15$ & $21.56 \pm 0.07$ \\
\hline BX-1071 & 123620.10 & +621112.6 & $\ldots / 1.996$ & UV & $<31.8$ & $\ldots$ & & $23.06 \pm 0.21$ \\
\hline BX-1228 & 123620.19 & +621540.6 & $1.999 / 1.995$ & UV & $<14.1$ & $14.7 \pm 4.1$ & $23.05 \pm 0.22$ & $23.10 \pm 0.11$ \\
\hline BX-1267 & 123622.67 & +621621.6 & $1.996 / 1.996$ & UV & $<14.5$ & $48.7 \pm 7.2$ & & 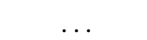 \\
\hline BX-1339 & 123625.09 & +621756.8 & $1.993 / 1.984$ & UV & $<14.9$ & $\ldots$ & $22.63 \pm 0.31$ & $23.03 \pm 0.12$ \\
\hline BX-1307 & 123648.33 & +621416.7 & $\ldots / 2.002$ & UV & $18.2 \pm 6.4(5.2)$ & $145.5 \pm 12.2$ & $21.34 \pm 0.09$ & $21.61 \pm 0.07$ \\
\hline BX-1329 & 123654.62 & +621407.7 & $\ldots / 1.987$ & UV & $<18.2$ & $\ldots$ & $\ldots$ & $\ldots$ \\
\hline BX-1827 & 123656.63 & +621519.0 & $1.988 / \ldots$ & UV & $9.6 \pm 4.1(2.3)$ & $\ldots$ & $\ldots$ & $23.46 \pm 0.13$ \\
\hline BX1129 & 123656.94 & +620848.7 & $\ldots / 1.973$ & UV & $21.7 \pm 11.2(3.0)$ & $104.4 \pm 10.3$ & $21.46 \pm 0.09$ & $21.60 \pm 0.07$ \\
\hline BX-1431 & 123658.48 & +621645.5 & $2.006 / 1.996$ & UV & $<13.4$ & $\ldots$ & $\ldots$ & $23.25 \pm 0.34$ \\
\hline BX1378 & 123702.02 & +62 1443.4 & $\ldots / 1.971$ & UV & $10.4 \pm 4.2(2.5)$ & $29.4 \pm 5.5$ & $22.43 \pm 0.10$ & 22.400 .07 \\
\hline BM-1122 & 123702.62 & +621156.7 & $1.994 / 1.986$ & UV & $<17.6$ & $\ldots$ & $\ldots$ & $23.30 \pm 0.16$ \\
\hline BX-1434 & 123716.80 & +621438.8 & $\ldots / 1.994$ & UV & $4.6 \pm 6.1(1.5)$ & $\ldots$ & $\ldots$ & $23.18 \pm 0.27$ \\
\hline BM-69 & 123717.68 & +62 1435.9 & $1.991 / 1.991$ & UV & $<17.8$ & $32.7 \pm 5.8$ & $\ldots$ & $\ldots$ \\
\hline BX-184 & 123719.28 & +621300.6 & $1.998 / \ldots$ & UV & $<12.2$ & $\ldots$ & $\ldots$ & $22.95 \pm 0.25$ \\
\hline BM-70 & 123720.05 & +621457.1 & $1.997 / 1.994$ & UV & $<30.9$ & $\ldots$ & $\ldots$ & $23.69 \pm 0.19$ \\
\hline BX-1605 & 123721.51 & +621830.6 & $1.977 / 1.970$ & UV & $<23.1$ & $\ldots$ & $\ldots$ & $23.36 \pm 0.17$ \\
\hline BM-1155 & 123723.98 & +621212.1 & $2.024 / 2.015$ & UV & $<33.6$ & $\ldots$ & $\ldots$ & $23.28 \pm 0.13$ \\
\hline BX-1642 & 123732.40 & +621750.8 & $2.010 / 2.004$ & UV & $<21.0$ & $\ldots$ & $\ldots$ & $22.95 \pm 0.09$ \\
\hline BX-1708 & 123732.65 & +621910.6 & $\ldots / 1.987$ & UV & $16.3 \pm 9.1(3.0)$ & $208.3 \pm 14.6$ & $21.03 \pm 0.07$ & $21.23 \pm 0.07$ \\
\hline BX-1694 & 123733.82 & +621846.3 & $2.009 / 2.005$ & UV & $10.1 \pm 8.4(2.0)$ & $13.8 \pm 4.3$ & $22.28 \pm 0.11$ & $22.58 \pm 0.07$ \\
\hline SCBX-6106 & 123710.82 & +620819.0 & $\ldots / 1.984$ & UV & $<13.5$ & $\ldots$ & $22.41 \pm 0.55$ & $23.15 \pm 0.22$ \\
\hline SCBX-6946 & 123657.70 & +621036.8 & $\ldots / 1.992$ & UV & $<14.7$ & $\ldots$ & $22.53 \pm 0.31$ & $22.84 \pm 0.25$ \\
\hline
\end{tabular}

Notes.

${ }^{a}$ Galaxies listed have redshifts that lie within $\pm 2 \sigma$ of the $z=1.99$ peak, and as shown by the vertical bars in Figure $2 \mathrm{~b}$.

${ }^{\mathrm{b}}$ Redshifts of UV-selected galaxies are quoted as emission line/absorption line, where "..." indicates that one or the other was not possible from the spectrum — see Reddy et al. (2006) for details of the BX/BM galaxies, whereas we have adopted this same procedure for the SCBX galaxies (from our own redshift surveys), selected from a color-cut transformed to AB-mag from that presented in Steidel et al. (2004). Molecular gas (CO) redshifts are from Chapman et al. (2008), and I. Smail et al. (2009, in preparation). The Spitzer-IRS redshift comes from Pope et al. (2008).

${ }^{c}$ Radio fluxes from Morrison et al. (2008), derived from the full resolution ( $\left.1^{\prime \prime} .7\right)$ map. Integrated flux measurements and errors are shown, with the signal-to-noise ratio $(\mathrm{S} / \mathrm{N})$ in brackets. For UV galaxies, source fluxes are listed if a radio peak lies within $0.5^{\prime \prime}$ of the optical source, otherwise a $3 \sigma$ upper limit.

d $24 \mu \mathrm{m}$ flux density of SMG-H140w is confused with SMG-H140e, although the radio emission is clearly resolved by MERLIN (Chapman et al. 2004b), and even by the VLA (Biggs \& Ivison 2006).

${ }^{\mathrm{e}} \mathrm{HDF} 172$ could conceivably be interpreted as an SFRG rather than an SMG. It is a SCUBA photometry detection in Chapman et al. (2001), a SCUBA-mapping nondetection in Borys et al. (2004), and a weak $(\sim 3 \sigma)$ MAMBO-mapping source (at $1.2 \mathrm{~mm}$ ) from Greve et al. (2008).

$\delta_{g}^{z}=n_{z} /(n-1)=2.5$. While C05 showed that many SMGs do have the colors of UV-selected galaxies, these spike SMGs and SFRGs do not overlap at all with the UV-selected galaxies in this $z \sim 1.99$ structure.

We have therefore identified a highly significant overdensity in ultraluminous SMGs, which appears not to correspond to a similarly overdense structure in less luminous, UV-selected galaxies. The UV galaxies have much lower SFRs and longer lifetimes (Reddy et al. 2006) than SMGs, and it is a reasonable hypothesis that the UV-population would represent a more dependable tracer of large-scale structures. While there remains the possibility that redshift incompleteness in quiescent galaxy populations (for example) could be responsible for the discrepancy, we will proceed with our explicit findings of a different bias between the two star-forming galaxy populations.

\subsection{Analysis of the $z=1.99$ Spike Galaxies}

The environmental context of high-redshift galaxy clusters is a topic that has lately received interest, since the preferential buildup of stellar mass in clusters should happen at earlier epochs than for field galaxies. Steidel et al. (2005) found considerable excess stellar mass buildup and longer mean stellar ages in the densest structure from their $z \sim 2$ surveys, as compared with the coeval field population. Clustering results from large spectroscopic surveys (e.g., VVDS; Meneux et al. 2008), rest- $K$ mass-selected surveys using the Infrared Array Camera (IRAC) on Spitzer (e.g., de La Torre et al. 2007), and red galaxy selection (e.g., BzK/extremely red object (ERO) galaxies; Daddi et al. 2003) have come to similar conclusions regarding these environmental dependences. Our naive explanation of the 

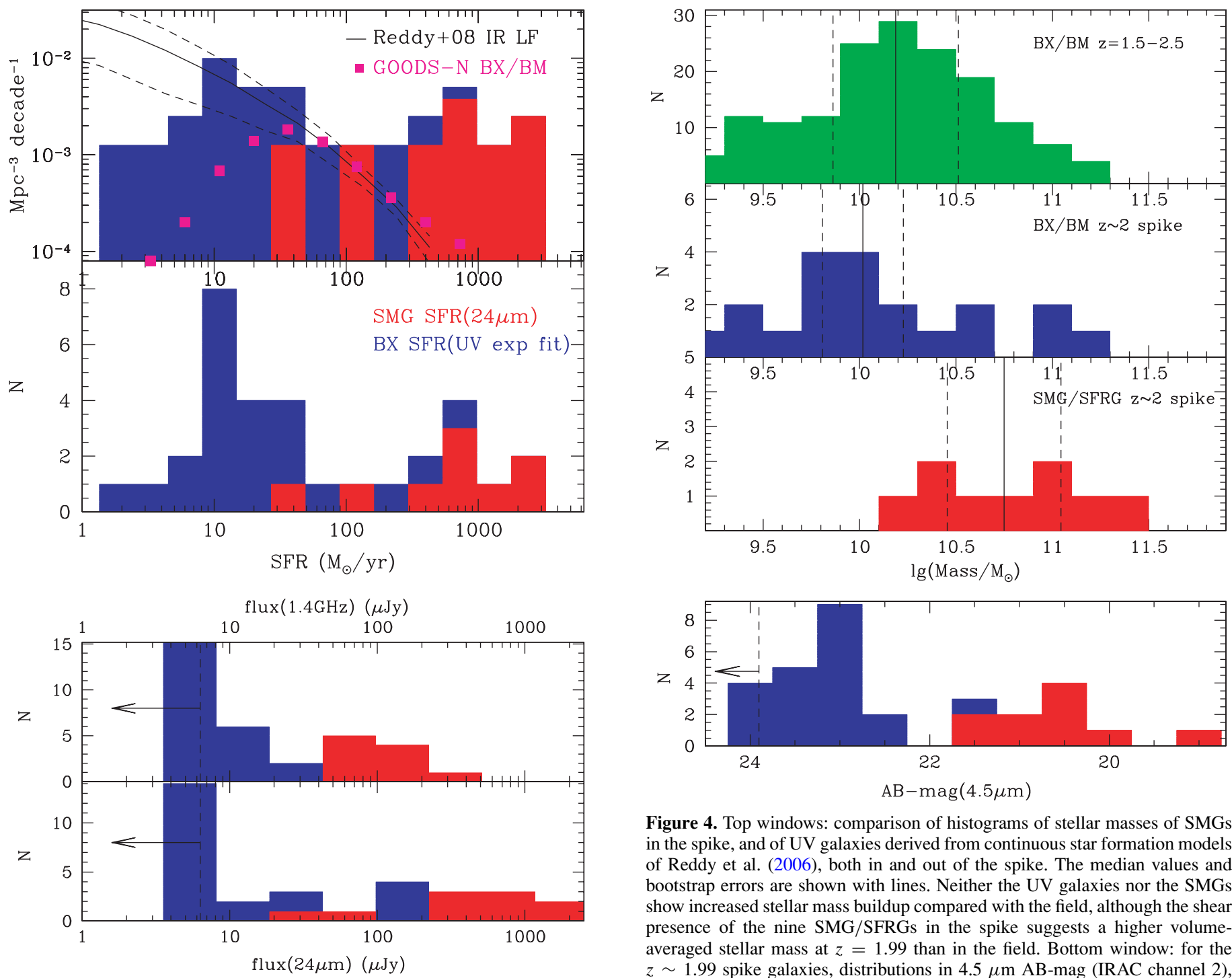

Figure 4. Top windows: comparison of histograms of stellar masses of SMGs in the spike, and of UV galaxies derived from continuous star formation models of Reddy et al. (2006), both in and out of the spike. The median values and bootstrap errors are shown with lines. Neither the UV galaxies nor the SMGs show increased stellar mass buildup compared with the field, although the shear presence of the nine SMG/SFRGs in the spike suggests a higher volumeaveraged stellar mass at $z=1.99$ than in the field. Bottom window: for the $z \sim 1.99$ spike galaxies, distributions in $4.5 \mu \mathrm{m} \mathrm{AB-mag} \mathrm{(IRAC} \mathrm{channel} \mathrm{2),}$ representing a rest frame $\sim 1.6 \mu \mathrm{m}$ or $H$-band light, showing the same trend as the stellar mass calculated above from the complete spectral energy distributions (SEDs).

LF for $z=1.9-2.7$ (translated into SFR using Kennicutt 1998) to the SFR for SMGs (red) derived from $24 \mu \mathrm{m}$, and for UV-selected galaxies derived from exponentially declining star formation models (blue). The IR LF of just the UV-selected galaxies in GOODS-N is also shown (not corrected for completeness) as squares, for direct comparison to the spike galaxies (which also are not corrected for completeness at the low-luminosity end). As expected, the vast majority of the overdensity compared to the average field appears at the high-SFR end in SMGs. Below is shown the raw distributions in SFR for UV galaxies and SMGs/SFRGs. Lower windows: for the $z \sim 1.99$ spike galaxies, distributions in radio $(1.4 \mathrm{GHz})$ flux and $24 \mu \mathrm{m}$ flux density are presented, to show consistent measures across UV-selected and SMG/SFRG populations, unaffected by uncertainties in SFRs calculated by different methods.

(A color version of this figure is available in the online journal.)

differential bias between the submillimeter and UV-selected galaxies invokes a modest mass cluster in an active formation period, and we might therefore expect to find little in the way of stellar mass or age differences in the spike population. However, our interpretation could be clouded by missing populations of evolved galaxies, which are not well sampled by GOODS-N spectroscopic surveys. We therefore analyze the spike galaxies to see if they show environmental effects characteristic of other high- $z$ clusters.

First, we look at SFRs for all the galaxies in the spike (Figure 3). Since many of the UV-selected galaxies are not detected in the $24 \mu \mathrm{m}$ or radio bands, while the SMGs and SFRGs typically have their SFRs underestimated by large

factors in the UV, we calculate SFRs for the UV galaxies from the dust-corrected UV luminosity, and from the $24 \mu \mathrm{m}$ luminosity for the SMGs and SFRGs. For the spike galaxies we also show the distributions of radio $(1.4 \mathrm{GHz})$ and $24 \mu \mathrm{m}$ flux density to demonstrate that our derived SFRs are consistent with the raw data. The clustered environment allows for a useful comparison of SFR indicators without $K$-correction and model galaxy uncertainties. Our new radio measurements for the spike galaxies detect significant numbers of the lower luminosity UVselected galaxies in addition to all the SMGs and SFRGs, and radio-derived SFRs are generally consistent with those derived from Spitzer $24 \mu \mathrm{m}$ fluxes. We then consider the LF of the whole $z \sim 1.99$ spike and compare with the LF derived for the whole UV population by Reddy et al. (2006) in Figure 3. As expected for a relatively insignificant structure in the UV, the Reddy et al. LF is reasonably consistent with the $z \sim 2$ field galaxies, with a small excess expected from the overdensity factor of order 2. When added to the spike sample, the SMGs clearly form a significant excess compared with the average LF of all (dust-corrected) UV-selected galaxies. 
Next, we consider the stellar masses of galaxies in the spike (Figure 4), compared to all nonspike UV-selected galaxies. The UV galaxies in the structure do not exhibit the enhanced buildup of stellar mass, which is apparent in other high- $z$ UV/opticalselected clusters (Steidel et al. 2005; Kurk et al. 2008). The UV spike galaxies have $\left\langle M^{*}\right\rangle=1.1 \pm 3.6 \times 10^{10} M_{\odot}$, while the "field" UV galaxies have $\left\langle M^{*}\right\rangle=1.5 \pm 3.4 \times 10^{10} M_{\odot}$ (derived from the tables in Reddy et al. 2006). Using the stellar masses estimated for SMGs by Hainline (2008) and L. J. Hainline et al. (2009, in preparation), we find that the spike SMGs clearly have larger stellar masses $\left(\left\langle M^{*}\right\rangle=6.0 \pm\right.$ $3.6 \times 10^{10} M_{\odot}$ ) than the UV-selected galaxies, but the SMGs' stellar masses are not significantly different from the median for 73 SMGs from Hainline $\left(2008 ; M^{*}=6.9 \times 10^{10} M_{\odot}\right)$. We note that Borys et al. (2005) derived even larger $(5 \times)$ stellar masses for these spike SMGs. Hainline (2008) ascribe the difference in their masses to those from Borys et al. as due to two issues. First, the use of different stellar evolution models: the Maraston TP-AGB models (Maraston et al. 2006), and differing light-to-mass and star formation (SF) histories, and second, the removal of AGN contributions to the observed $8 \mu \mathrm{m}$ flux prior to fitting. Alexander et al. (2008) have also explicitly attempted to correct for the AGN contribution to the rest-frame $K$-band light in these SMGs and derive lower masses (a factor of $\sim 2$ ). We stress that the relative masses of the BX/ BMs and SMGs will be robust against some of the systematic uncertainties in the models, and that our results are not sensitive to changing the masses by a few factors, and hence we believe that our conclusions are robust. We also show in Figure 4 the $4.5 \mu \mathrm{m}$ fluxes of the spike galaxies (rest frame $\sim 1.6 \mu \mathrm{m}$ or $H$-band) revealing the SMGs are clearly much brighter than the UV galaxies without uncertainties in stellar mass model fitting. There is therefore evidence for significant stellar buildup in our $z \sim 1.99$ overdense environment, although this is driven exclusively by the large overdensity of SMGs.

Comparing our "spike" SMGs to "typical" SMGs at similar epochs (e.g., Chapman et al. 2005; Borys et al. 2005; Pope et al. 2006; Hainline 2008) reveals no obvious differences in SFR or stellar mass (the median values of both are similar for spike and field SMGs- SFR $\left.\rangle=810 M_{\odot} \mathrm{yr}^{-1},\left\langle M^{*}\right\rangle=7 \times 10^{10} M_{\odot}\right)$. An additional route to studying the activity in the structure versus the field would be through AGN populations. Cataloged AGNs in GOODS-N (e.g., Cowie et al. 2004; Reddy et al. 2006) do not reveal any significant enhancement at $z \sim 2$, nor do the X-ray properties of these spike SMGs (Alexander et al. 2005).

\section{DISCUSSION}

\subsection{Evolutionary Context of the Structure}

To place our SMG cluster in a cosmological context, we must assume a galaxy bias for SMGs. We adopt $b \sim 2.1$, a value consistent with simulations in van Kampen et al. (2005), and similar to the estimated galaxy bias in the UV galaxy sample (Adelberger et al. 2005a). Following the method introduced by Steidel et al. (1998), we relate the true mass overdensity $\delta$ to the observed redshift-space overdensity of SMGs $\delta_{g}^{z}$ through the expression

$$
1+b \delta=|C|\left(1+\delta_{g}^{z}\right),
$$

where $|C| \equiv V_{\text {app }} / V_{\text {true }}$ takes into account the effects of redshiftspace distortions from peculiar velocities. For our SMGs, SFRGs, and UV galaxies, $V_{\text {app }} \simeq 9900 \mathrm{Mpc}^{3}$ is the comoving volume in which the measurement is being made, bounded by the $10^{\prime} 0 \times 10^{\prime} 0$ region on the plane of the sky and the comoving distance, neglecting peculiar velocities, between $z=1.978$ and $z=2.008$. The volume correction factor can be approximated by $C=1+f-f(1+\delta)^{1 / 3}$ where $f=\Omega_{m}(z)^{0.6}=0.96$ at $z \simeq 2.0$. Thus $\delta \simeq 2.3$ with $\delta_{g}^{z}=10.0$, and $C=0.53$. The linear overdensity, $\delta_{0}$, can then be approximated using Equation (18) (spherical collapse) from Mo \& White (1996)

$$
\delta_{0} \simeq A_{1}(1+\delta)^{-2 / 3}+A_{2}(1+\delta)^{-0.5866}+A_{3}(1+\delta)^{-1 / 2}+A_{4}
$$

with $A_{1}=-1.35, A_{2}=0.78785, A_{3}=-1.12431$, and $A_{4}=1.68647$. Thus for our $z \sim 1.99 \mathrm{SMG}$ structure $(\delta=2.3)$, we find $\delta_{0} \simeq 0.85$ as the real space linear matter overdensity.

We now address what the SMG overdensity will become by redshift $z \sim 0$. Evolved forward in our adopted cosmology, its linear overdensity of $\delta_{0} \sim 0.85$ at $z=2$ corresponds to a linear overdensity of $\delta_{p} \sim 2.13$ at $z=0$. This exceeds the collapse threshold $\delta_{c}=1.69$.

However, if we repeat the analysis with the UV-selected galaxy $\delta_{\mathrm{UV}}^{z} \sim 2.5$ case, we find a matter overdensity at $z=2$ of only $\delta=0.83$, a linear overdensity $\delta_{0}=0.5$ or $\delta_{p} \sim 1.1$ at $z=0$, and the structure associated with this apparently modest overdensity of UV galaxies would not have collapsed by $z=0$.

The mass of a virialized object, as we would infer for the SMG overdensity, can be calculated by assuming that the SMG overdensity is associated with an Eulerian matter overdensity of $\delta_{m} \simeq 2.3$ at $z=2.0$ (e.g., Steidel et al. 1998), so its mass is $\left(1+\delta_{m}\right) \bar{\rho} V_{\text {true }} \sim 10^{15} M_{\odot}$ where $\bar{\rho}$ is the mean comoving matter density and $V_{\text {true }}=V_{\text {app }} / C \sim 18700 \mathrm{Mpc}^{3}$ is approximately the volume we calculated within the observed overdensity. There is, however, no straightforward way to estimate the mass of the lower contrast UV-galaxy spike, as it has not been obviously virialized by $z=0$. We simplistically assume that the inferred mass would be more than three times lower.

\subsection{Explaining the Differential Bias}

Our task is then to explain why such a significant structure in the SMG population exhibits such a modest overdensity in the UV-selected galaxies. One possibility is that the true overdensity and cluster mass concentration is in fact as large as implied by the SMGs, and we have missed a large population of evolved quiescent galaxies, selected for instance through the rest-frame $2 \mu \mathrm{m}$ emission. At a redshift $z \sim 2$, such a population is difficult to confirm spectroscopically, and might explain for instance why we found no difference in the stellar masses of field versus spike UV-selected star-forming galaxies. However, we see no logical reason why the mass concentration should then be represented by SMGs (and not UV galaxies), which are an even more luminous population of star-forming galaxies.

Another line of inquiry is to look for mechanisms to produce the apparent differential bias directly. Dynamical masses have been estimated for galaxies selected in the rest-frame UV using nebular emission line widths (Pettini et al. 2000; Erb et al. 2006), and for SMGs and SFRGs using both nebular lines and rotational CO molecular lines (e.g., Frayer et al. 1998; Neri et al. 2003; Swinbank et al. 2004, 2006, 2008; Chapman et al. 2008). Attempts to account for the fact that estimates of the dynamical masses of high- $z$ galaxies are significantly less than the clustering-inferred halo masses (Adelberger et al. 2005a; Blain et al. 2004) have fallen under the names of temporal bias (Scannapieco \& Thacker 2003), merger bias (e.g., Furlanetto \& Kamionkowski 2006), or assembly bias (Dalal et al. 2008). These approaches attempt to account for the rapid mergers and other properties of galaxies in halos boosting the apparent 
clustering signal, and they might naturally extend to the scenario we have uncovered in this $z \sim 2$ structure, which represents an even more radical version of this problem. As pointed out by Furlanetto \& Kamionkowski (2006), the extended PressSchechter and Mo \& White (1996) biasing scheme yields no merger bias as it explicitly ignores the variation of merger rates with the large-scale density field. However, for populations like SMGs, even an analytical approximation to the bias implicit in aggressively merging galaxies is suggestive that the clustering length could be enhanced. The challenge is to apply this formalism not just to a pairwise enhancement of the population, but to the synchronization of nine SMG-class galaxies spread over an angular region of $3.5 \mathrm{Mpc}$ on one side.

We consider from Furlanetto \& Kamionkowski (2006), Equation (24), which describes how pairs of peaks in the density field are clustered, as a function of the clustering of the peaks themselves (i.e., identifying the peaks with galaxies, and the "pair" as recently merged objects). In the limit that the peaks are well above the characteristic mass scale of objects (likely to be a reasonable assumption for SMGs), the correlation function of pairs $\chi(r)$ is

$$
\chi(r)=\left[1+\xi_{g}(r)\right]^{4}-1,
$$

where $\xi_{g}(r)$ is the correlation function of the galaxy population of interest ( $b_{g}$ is the associated matter correlation function). This gives an enhancement to the overall clustering of the underlying galaxy population.

Ignoring redshift space corrections, we then make the following argument: there is one true matter overdensity, $\delta_{m}$. Suppose that UV galaxies and SMGs are drawn from halos with the same mass (and hence bias $b_{\mathrm{UV}}$ ), then if SMGs correspond to pairs of objects, they have an extra "pair" bias $\left.b_{p}=\sqrt{(} \chi / \xi_{g}\right)$. So the observed overdensities $\delta^{z}$ will be

$$
\begin{gathered}
1+\delta_{\mathrm{UV}}^{z}=3.5=1+b_{\mathrm{UV}} \delta_{m} \\
1+\delta_{\mathrm{SMG}}^{z}=11=1+b_{p} b_{\mathrm{UV}} \delta_{m},
\end{gathered}
$$

which requires $b_{p} \sim 4$ (slightly different if redshift space correction is included).

While the model is approximate and the observations are not necessarily complete, the result is not unreasonable. A remaining question is what is the correct $r$ which applies in Equations (2) and (3) to evaluate $b_{p}$. On very large scales, where $\xi_{g} \ll 1$, we can Taylor expand Equation (3) about $r=0, \chi(r) \sim 4 \xi_{g}(r)$, to infer a pair bias $b_{p} \simeq 2$. Our physical interpretation of this is two pairs (or four SMGs), so we obtain four factors of $\xi_{g}$. However, on smaller scales, the pair bias goes up because one is dealing with peaks: on scales where the correlation function of SMGs is large, $\xi_{g} \sim 1$ (i.e., where clusters are forming), $\chi(r)=15$, so $b_{p} \sim 4$. This is similar to our required factor of boosting, and likely to be sufficient given uncertainties in the observations.

We emphasize that simulations that have tried to quantify this "assembly bias" give smaller effects, 10\% (Wechsler et al. 2002; Zhao et al. 2003a, 2003b; Dalal et al. 2008). However, these studies have not looked at this exact question of recent mergers, which may be a stronger effect than the concentration, age, spin, etc., which are modeled in these simulations, and therefore our toy model is not inconsistent with these studies.

\subsection{Context and Implications for SCUBA2 Surveys}

While the most overdense forming environments at $z>$ 1.5 have been observed to have large numbers of associated ultraluminous galaxies (e.g., the SSA22 $z=3.09$ protocluster has eight known SMGs; Geach et al. 2006), our results suggest that if one searches for galaxy clusters using the most vigorously bursting (and typically highest luminosity) systems, one is prone to finding the brief periods when systems of lower matter overdensity are rapidly evolving. The exceptionally strong concentration of SMGs presented here corresponds to a modest, not statistically significant (in terms of $\delta$ ) concentration in UV-selected galaxies, whereas the expectation would be that this submillimeter "spike" should represent one of the largest mass structures known. While we have outlined a toy model to explain the scenario in Section 3.2, it remains to be seen how such scenarios can be realized by detailed theoretical models and simulations of cluster formation.

Baugh et al. (2005) have presented a model of the SMG population in a semianalytical galaxy evolution framework. Using the Millennium Simulation (Springel et al. 2005), C. M. Almeida et al. (2009, in preparation) predict that the correlation function of model SMGs from Baugh et al. (2005) is close to a power law $\left(\eta=\left(r / r_{o}\right)^{\gamma}\right)$ over more than three decades in separation, with $\gamma=-1.94 \pm 0.05$ and a correlation length $r_{0}=8.8 \pm 0.3 \mathrm{Mpc}$, slightly smaller but consistent with that measured by Blain et al. (2004) from the redshifts of SMGs, $r_{0}=9.8 \pm 3.0 \mathrm{Mpc}$. Swinbank et al. (2008) performed a detailed comparison of actual SMG observations with model SMGs in the Baugh et al. (2005) model. In the Millennium Simulation, this correlation length corresponds to a predicted halo mass of $M_{\text {halo }}=3.1_{-1.9}^{+5.7} \times 10^{12} M_{\odot}$ (Gao et al. 2005). Measuring the dark halo mass for the model SMGs directly, Swinbank et al. (2008) derive $M_{\text {halo }}=3.6_{-1.5}^{+5.5} \times 10^{12} M_{\odot}$. Taken together this suggests that there is no merger bias in the correlation lengths: the correlation length of the SMGs is identical, within the errors, with the whole population of halos of the same masses. Our discovery in this paper of a large submillimeter burst in a modest mass environment suggests that a more thorough investigation is needed of the clustering properties of SMGs using combined semianalytical and numerical simulations to trace the star formation and merger histories of high-redshift galaxies.

We note that similar criticisms could apply to the clustering of AGN (e.g., Gilli et al. 2005), and overdensities selected through AGN (e.g., Rottgering et al. 1996, 2003; Brand et al. 2003). In particular, the overdensities of other galaxies around luminous AGNs are typically poorly characterized relative to the field. Our well calibrated results on SMGs suggest that these AGN-identified galaxy clusters could be far less massive than suggested.

While our characterization of a single cluster of SMGs cannot be extrapolated to cluster formation in general, it is noteworthy that large-scale surveys in the submillimeter with SCUBA2, and the Herschel and Planck space telescopes, and even the existing wide-field surveys at $24 \mu \mathrm{m}$ from Spitzer (e.g., Farrah et al. 2006) may reveal rather modest mass structures associated with some of the highest contrast source overdensities. The large beam size of Planck and the Herschel Spectral and Photometric Imaging REceiver (SPIRE) $500 \mu \mathrm{m}$ survey may be particularly sensitive to this sort of effect (Negrello et al. 2005, 2007), where the low-resolution surveys will measure the summed contributions of groups of sources within the beam. Further, the number of these low-mass but active clusters are likely to be significant enough to make it impossible to associate a large $r_{0}$ with a large dark-matter halo mass. Detailed follow-up of the highest concentrations of ultraluminous sources, although 
arduous, is clearly warranted. In the future, serendipitous searches for $\mathrm{CO}$ emitters at the same redshift using the Atacama Large Millimeter/Submillimeter Array (ALMA), and evidence for boosted flux from confused sources using Herschel may also provide insight into the nature of this structure.

\section{CONCLUSIONS}

We have described an apparent cluster of SMGs and RGs at $\langle z\rangle=1.99$ in the GOODS-N field, the strongest known association of SMGs (Blain et al. 2004; Chapman et al. 2005). The implied matter overdensity from the redshift space contrast $\delta_{g}^{z} \simeq 10$ is $\sim 10^{15} M_{\odot}$. Searching the same volume in UVselected galaxies, we find a much reduced overdensity $\left(\delta_{g}^{z} \simeq\right.$ $2.5)$. The low SFRs of UV galaxies and much longer star formation timescales on average compared with the SMGs and SFRGs suggest they should be more dependable for defining the mass scale of this structure. We conclude that we are observing a modest mass overdensity (which likely will not even virialize by $z=0$ ), which is undergoing a particularly active star formation phase.

We have characterized the bolometric LF of the $z \sim 1.99$ spike galaxies compared to that estimated for a large sample of UV-selected star-forming galaxies (Reddy et al. 2008), finding that it is almost entirely in the SMG population where an excess is seen. The clustered environment allows for a useful comparison of SFR and stellar mass indicators without $K$-correction and model galaxy uncertainties. Our new radio measurements for the spike galaxies detect significant numbers of the lower luminosity UV-selected galaxies in addition to all the SMGs and SFRGs, and radio-derived SFRs are generally consistent with those derived from Spitzer $24 \mu \mathrm{m}$ fluxes.

While the most overdense forming environments at $z>1.5$ have been observed to have large numbers of associated ultraluminous galaxies, our results here suggest that if one searches for galaxy clusters using the highest luminosity systems, one is prone to find the brief periods when systems of lower matter overdensity are rapidly evolving. SMGs do not obviously trace the most massive structures in the universe, as simulations and constraints to date have suggested they should. The success of our toy model with merger bias explaining the basic elements of our observations suggests that simulations may not currently be accurately reflecting the processes occurring in the formative phases of galaxy clusters. Further work to embed these aspects of merger bias in simulations will therefore be of great interest in understanding galaxy cluster assembly. Investigation of similar systems in upcoming surveys at long wavelengths will be of great interest to explore the full range of environments associated with strong overdensities of ultraluminous galaxies.

We thank Steve Furlanetto for very helpful discussions on extending his work on merger bias to our SMG cluster, as well as the anonymous referee for his suggestions. S.C.C. acknowledges an NSERC Discovery grant and a fellowship from the Canadian Space Agency which supported some of this work. I.R.S. acknowledges support from the Royal Society.

\section{REFERENCES}

Adelberger, K., et al. 2005a, ApJ, 619, 697

Adelberger, K., et al. 2005b, ApJ, 621, 574

Alexander, D., Bauer, F., Chapman, S. C., Smail, I., Blain, A., Brandt, N., \& Ivison, R. 2005, ApJ, 632, 736
Alexander, D., et al. 2008, AJ, 135, 1968

Almaini, O., Lawrence, A., \& Boyle, B. J. 1999, MNRAS, 305L, 59

Baugh, C. M., Lacey, C. G., Frenk, C. S., Granato, G. L., Silva, L., Bressan, Benson, A. J., \& Cole, S. 2005, MNRAS, 356, 1191

Biggs, A., \& Ivison, R. 2006, MNRAS, 371, 963

Biggs, A., \& Ivison, R. 2008, MNRAS, 385, 893

Blain, A. W., Chapman, S., Smail, I., \& Ivison, R. J. 2004, ApJ, 611, 725

Blain, A. W., Smail, I., Ivison, R. J., Kneib, J.-P., \& Frayer, D. T. 2002, Phys. Rep., 369, 111

Blake, C., Pope, A., Scott, D., \& Mobasher, B. 2006, MNRAS, 368, 732

Borys, C., Chapman, S. C., Halpern, M., \& Scott, D. 2003, MNRAS, 355,485

Borys, C., Scott, D., Chapman, S. C., Halpern, M., Pope, A., \& Nandra, K. 2004, MNRAS, 355, 485

Borys, C., Smail, I., Chapman, S. C., Blain, A. W., Alexander, D. M., \& Ivison, R. J. 2005, ApJ, 635, 853

Brand, K., Rawlings, S., Hill, G., Lacy, M., Mitchell, E., \& Tufts, J. 2003, MNRAS, 344, 283

Brand, K., Rawlings, S., Hill, G., \& Tufts, J. 2005, MNRAS, 357, 1231

Brown, M., Jannuzi, B., Dey, A., \& Tiede, G. 2005, ApJ, 621, 41

Casey, C. 2008, MNRAS, submitted

Chapman, S. C., Blain, A., Ivison, R., \& Smail, I. 2003, Nature, 422, 695

Chapman, S. C., Blain, A., Smail, I., \& Ivison, R. 2005, ApJ, 622, 772

Chapman, S. C., Ibata, R., Lewis, G., Ferguson, A., Irwin, M., McConnachie, A., \& Tanvir, N. 2006, ApJ, 653, 255

Chapman, S. C., Richards, E., Lewis, G., Wilson, G., \& Barger, A. 2001, ApJ, $548,147 \mathrm{~L}$

Chapman, S. C., Smail, I., Blain, A., \& Ivison, R. 2004b, ApJ, 614, 671

Chapman, S. C., Smail, I., Windhorst, R., Muxlow, T., \& Ivison, R. 2004a, ApJ, 611,732

Chapman, S. C., et al. 2000, MNRAS, 319, 318

Chapman, S. C., et al. 2008, ApJ, 689, 889

Coppin, K., et al. 2008, MNRAS, 384, 1597

Cowie, L., et al. 2004, AJ, 127, 3137

Daddi, E., et al. 2003, ApJ, 588, 50

Daddi, E., et al. 2008, ApJ, in press, arXiv:0810.3108

Dalal, N., White, M., Bond, J. R., \& Shirokov, A. 2008, ApJ, 687, 12

de Breuck, C., Rottgering, H., Miley, G., van Breugel, W., \& Best, P. 2000, A\&A, 362, 519

de La Torre, S., et al. 2007, A\&A, 475, 443

Eadie, W. T., Drijard, D., James, F. E., Roos, M., \& Sadoulet, B. 1971, Statistical Methods in Experimental Physics (Amsterdam: North-Holland)

Erb, D., et al. 2006, ApJ, 646, 107

Fabian, A. C., et al. 2000, MNRAS, 315L, 8

Farrah, D., et al. 2006, ApJ, 641, 17L

Frayer, D., et al. 1998, ApJ, 506, L7

Furlanetto, S., \& Kamionkowski, M. 2006, MNRAS, 366, 529

Gao, L., Springel, V., \& White, S. D. M. 2005, MNRAS, 363, L66

Geach, J. E., et al. 2006, ApJ, 649, 661

Gilli, R., et al. 2005, A\&A, 430, 811

Gilli, R., et al. 2007, A\&A, 475, 83

Greve, T. R., et al. 2005, MNRAS, 359, 1165

Greve, T. R., Pope, A., Scott, D., Ivison, R. J., Borys, C., Conselice, C. J., \& Bertoldi, F. 2008, MNRAS, 389, 1489

Hainline, L. J. 2008, PhD thesis, Caltech

Ivison, R. J., Dunlop, J. S., Smail, I., Dey, A., Lui, M. C., \& Graham, J. R. 2000, ApJ, 542, 27

Kennicutt, R. C., Jr. 1998, ApJ, 498, 541

Knudsen, K. K., van der Werf, P. P., \& Kneib, J.-P. 2008, MNRAS, 384, 1611

Kurk, J., et al. 2008, arXiv:0804.4126

Maraston, C., Daddi, E., Renzini, A., Cimatti, A., Dickinson, M., Papovich, C., Pasquali, A., \& Pirzkal, N. 2006, ApJ, 652, 85

Menendez-Delmestre, K., et al. 2007, ApJ, 655, L65

Meneux, B., et al. 2008, A\&A, 478, 299

Mo, H. J., \& White, S. D. M. 1996, MNRAS, 282, 347

Morrison, G., et al. 2008, in ASP Conf. Ser. 381, Infrared Diagnostics of Galaxy Evolution, ed. R.-R. Chary, H. I. Teplitz, \& K. Sheth (San Francisco, CA: ASP), 376

Negrello, M., Gonzalez-Nuevo, J., Magliocchetti, M., Moscardini, L., De Zotti, G., Toffolatti, L., \& Danese, L. 2005, MNRAS, 358, 869

Negrello, M., et al. 2007, MNRAS, 377, 1557

Neri, R., et al. 2003, ApJ, 597, L113

Pentericci, L., Kurk, J. D., Carilli, C. L., Harris, D. E., Miley, G. K., \& Röttgering, H. J. A. 2002, A\&A, 396, 109

Pettini, M., Steidel, C. C., Adelberger, K. L., Dickinson, M., \& Giavalisco, M. 2000, ApJ, 528, 96 
Pope, A., et al. 2006, MNRAS, 370, 1185

Pope, A., et al. 2008, ApJ, 675, 1171

Reddy, N. A., Steidel, C. C., Fadda, D., Yan, L., Pettini, M., Shapley, A. E., Erb,

D. K., \& Adelberger, K. L. 2006, ApJ, 644, 792

Reddy, N. A., et al. 2008, ApJS, 175, 48

Rottgering, H. J. A., West, M. J., Miley, G. K., \& Chambers, K. C. 1996, A\&A, 307,376

Rottgering, H. J. A., et al. 2003, NewAR, 47, 309

Scannapieco, E., \& Thacker, R. J. 2003, ApJ, 590, 69L

Scott, S. E., Dunlop, J. S., \& Serjeant, S. 2006, MNRAS, 370, 1057

Scott, S. E., et al. 2002, MNRAS, 331, 817

Smail, I., Irison, R. J., \& Blain, A. W. 1997, ApJ, 490, 5L

Smail, I., et al. 2003a, ApJ, 599, 86

Smail, I., et al. 2003b, MNRAS, 342, 1185

Spergel, D. N., et al. 2003, ApJS, 148, 175

Springel, V., et al. 2005, Nature, 435, 629

Steidel, C. C., Adelberger, K. L., Dickinson, M., Giavalisco, M., Pettini, M., \& Kellogg, M. 1998, ApJ, 492, 428
Steidel, C. C., Adelberger, K. L., Shapley, A. E., Erb, D. K., Reddy, B. A., \& Pettini, M. 2005, ApJ, 626, 44

Steidel, C. C., Shapley, A. E., Pettini, M., Adelberger, K. L., Erb, D. K., Reddy, N. A., \& Hunt, M. P. 2004, ApJ, 604, 534

Stevens, J. A., et al. 2003, Nature, 425, 264

Swinbank, A. M., Chapman, S. C., Smail, I., Lindner, C., Borys, C., Blain, A W., Ivison, R. J., \& Lewis, G. F. 2006, MNRAS, 371, 465

Swinbank, A. M., Smail, I., Chapman, S. C., Blain, A. W., Ivison, R. J., \& Keel, W. C. 2004, ApJ, 617, 64

Swinbank, A. M., et al. 2008, MNRAS, 391, 420

Tacconi, L. J., et al. 2006, ApJ, 640, 228

Tacconi, L. J., et al. 2008, ApJ, 680, 246

van Kampen, E., et al. 2005, MNRAS, 359, 469

Wechsler, R. H., Bullock, J. S., Primack, J. R., Kravtsov, A. V., \& Dekel, A 2002, ApJ, 568, 52

Wirth, G., et al. 2004, AJ, 127, 3121

Zhao, D. H., Jing, Y. P., Mo, H. J., \& Borner, G. 2003a, ApJ, 597, L9

Zhao, D. H., Mo, H. J., Jing, Y. P., \& Borner, G. 2003b, MNRAS, 339, 12 